TRANSACTIONS OF THE

AMERICAN MATHEMATICAL SOCIETY

Volume 354, Number 9, Pages 3743-3755

S 0002-9947(02)03017-9

Article electronically published on April 23, 2002

\title{
HOMOGENEOUS WEAK SOLENOIDS
}

\author{
ROBBERT FOKKINK AND LEX OVERSTEEGEN
}

\begin{abstract}
A (generalized) weak solenoid is an inverse limit space over manifolds with bonding maps that are covering maps. If the covering maps are regular, then we call the inverse limit space a strong solenoid. By a theorem of M.C. McCord, strong solenoids are homogeneous. We show conversely that homogeneous weak solenoids are topologically equivalent to strong solenoids. We also give an example of a weak solenoid that has simply connected pathcomponents, but which is not homogeneous.
\end{abstract}

\section{INTRODUCTION}

A (one-dimensional) solenoid is an inverse limit space of circles $S^{1}=\{z \in$ $\mathbb{C}:|z|=1\}$, with bonding maps $p(z)=z^{n}$ for some $n \in \mathbb{N}$. Solenoids were introduced in 1928 by van Dantzig and van der Waerden 8 . Solenoids have topological structure, being indecomposable continua, and solenoids have algebraic structure, being compact abelian groups. In this paper we shall study higher-dimensional generalizations of solenoids that were first studied by M.C. McCord [15. We shall be interested in the conditions that make a higher-dimensional solenoid $\mathcal{S}$ homogeneous, i.e., for each pair of points $x, y \in \mathcal{S}$ there exists a homeomorphism that maps $x$ to $y$. This interest is motivated by Hagopian's topological characterization of solenoids.

Theorem 1 (Hagopian, [10]). A homogeneous metric continuum is a solenoid if and only if every proper subcontinuum is an arc.

In this characterization, the circle is a solenoid for the degenerate case in which the bonding map is the identity. This result was conjectured by Bing [5]. Later, different proofs were given in [18] and [2]. Both these proofs concern the local product structure of a homogeneous one-dimensional space and, as a byproduct, yield another characterization.

Theorem 2. A homogeneous metric continuum is a solenoid if and only if it has a local product structure of a Cantor set cross an arc.

In this characterization, the circle is not a solenoid. The advantage of this second characterization is that it can be extended to higher dimensions for inverse limit spaces over covering maps of manifolds. We shall call such spaces weak solenoids and defer their exact definition to the next section. Schori [22] showed that weak

Received by the editors April 4, 2001 and, in revised form, January 4, 2002.

2000 Mathematics Subject Classification. Primary 54F15, 57M10; Secondary 54C10, 55R10.

Key words and phrases. Homogeneous continuum, solenoid, covering space, profinite group, principal bundle.

The second author was supported in part by NSF-DMS-0072626. 
solenoids need not be homogeneous. On the other hand, McCord [15] derived a criterion for weak solenoids to be homogeneous. We shall say that an inverse limit space is a strong solenoid if it satisfies McCord's criterion. The main result of our paper reads as follows.

Theorem 3. If a weak solenoid is homogeneous, then it is homeomorphic to a strong solenoid.

This theorem is a modest first step towards a proof of the following conjecture, which presents itself quite naturally from results of Alex Clark [6]. It generalizes Theorem 2

Conjecture 4. A homogeneous metric continuum is a strong solenoid if and only if it has a local product structure of a Cantor set cross an $n$-cell.

We remark that such a local structure also occurs in the work of Williams 27 on hyperbolic attractors.

Our main result settles Question 1 of 21. Question 2 in that paper asks whether a weak solenoid is homogeneous if, and only if, all its path components are homeomorphic. We shall not settle this question; however, we give an example of a non-homogeneous weak solenoid with simply-connected path-components. Results of Ronald de Man [13] indicate that such components are all homeomorphic.

\section{WEAK SOLENOIDS}

A weak solenoid is an inverse limit sequence $\lim _{\leftarrow}\left(M_{i}, p^{i}\right)$ over compact and closed manifolds $M_{i}$ such that each bonding map $p^{i}: M_{i} \rightarrow M_{i-1}$ is a covering map. The composition $p^{i} \circ p^{i-1} \circ \ldots \circ p^{j+1}$ is denoted by $p_{j}^{i}: M_{i} \rightarrow M_{j}$. We shall call the inverse limit space a strong solenoid if any finite composition of the bonding maps $p_{j}^{i}$ is a regular covering map. The covering map $p_{0}^{i}: M_{i} \rightarrow M_{0}$ shall be denoted by $p_{i}$. Recall that a covering map $p:(M, m) \rightarrow(N, n)$ is regular if $p_{*}\left(\pi_{1}(M, m)\right)$ is a normal subgroup of $\pi_{1}(N, n)$, where $p_{*}$ denotes the induced homomorphism between the fundamental groups.

Theorem 5 (McCord, [15]). A strong solenoid is homogeneous.

Schori [22] gave an example of a weak solenoid that is not homogeneous, thus settling a problem of J. Segal [24].

Theorem 6 (Schori). There exist weak solenoids that are not homogeneous.

A different example was later given by Rogers and Tollefson 19. We shall review both examples in this paper. Schori conjectured that McCord's condition was both necessary and sufficient.

Conjecture 7 (Schori). If a weak solenoid $\lim _{\leftarrow}\left(M_{i}, p^{i}\right)$ is homogeneous, then for some index $j$ all composite projections $p_{j}^{i}: M_{i} \rightarrow M_{j}$ are regular.

This conjecture was shown to be false by Rogers and Tollefson [20. However, as Theorem 3 shows, Schori's conjecture is true under a slight modification, as suggested by Question 1 in [20]. 


\section{Properties of COVERING SPACES AND COVERING MAPS}

The spaces under consideration are compact, connected manifolds without boundary and with an infinite fundamental group. We summarize some standard properties of covering spaces and covering maps, for which [14] is a general reference.

Theorem 8. Suppose that $p: M \rightarrow N$ is a covering map and that $f, g: X \rightarrow M$ are continuous. If $p \circ f=p \circ g$ and $f(x)=g(x)$ for some $x \in X$, then $f=g$.

In our case, $f$ and $g$ shall be continuous maps between pointed spaces, so that the condition $f(x)=g(x)$ is satisfied by the base point.

Theorem 9 (Lifting property). Suppose that $p:(M, m) \rightarrow(N, n)$ is a covering map and that $f:(K, k) \rightarrow(N, n)$ is a continuous map. Then there exists a lift $\tilde{f}:(K, k) \rightarrow(M, m)$, i.e., $p \circ \tilde{f}=f$, if and only if $f_{*}\left(\pi_{1}(K, k)\right) \subset p_{*}\left(\pi_{1}(M, m)\right)$.

The fiber of a covering map $p:(M, m) \rightarrow(N, n)$ corresponds to the (right) residue classes of $p_{*} \pi_{1}(M, m)$ in $\pi_{1}(N, n)$. Denote $H=p_{*} \pi_{1}(M, m)$ and $G=$ $\pi_{1}(N, n)$. Then $G$ acts on the fiber by right multiplication of the (right) residue classes $G / H$.

A deck transformation $f: M \rightarrow M$ is a map preserves the fibers of $N$. Deck transformations thus form a group that acts on a fiber. The action is transitive if and only if the covering map is regular.

For non-pointed spaces the lifting property extends as follows.

Theorem 10 (Homotopy lifting property). Suppose that $p: M \rightarrow N$ is a covering map and that $F: K \times[0,1] \rightarrow N$ is a homotopy. For some $x_{0} \in K$ let $\gamma:[0,1] \rightarrow N$ be the path defined by $\gamma(t)=F\left(x_{0}, t\right)$ and let $\tilde{\gamma}$ be a path in $M$ covering $\gamma$. If the restriction of $F$ to $K \times\{0\}$ can be lifted, then the entire homotopy can be lifted to a homotopy $\tilde{F}$, such that $\tilde{F}\left(x_{0}, t\right)=\tilde{\gamma}(t)$.

If no confusion is likely to arise, we shall denote a weak solenoid $\lim _{\leftarrow}\left(M_{i}, p^{i}\right)$ by $M_{\infty}$. A point $x \in M_{\infty}$ then represents a sequence $x_{i} \in M_{i}$. We review some elementary properties of weak solenoids.

Lemma 11. Suppose that $M_{\infty}$ is a weak solenoid and that $x, y \in M_{\infty}$. Then there exists a path $\gamma:[0,1] \rightarrow M_{\infty}$ such that $\gamma(0)=x$ and such that $z=\gamma(1)$ has its first coordinate in common with $y$, i.e., $z_{0}=y_{0}$.

Proof. Define a path $\gamma_{0}:[0,1] \rightarrow M_{0}$ with beginning point $x_{0}$ and end point $y_{0}$. By Theorem 9 there exists a lift $\gamma_{i}:([0,1], 0) \rightarrow\left(M_{i}, x_{i}\right)$ for every $i \in \mathbb{N}$. Now define $\gamma:[0,1] \rightarrow M_{\infty}$ by $\gamma(t)=\left(\gamma_{i}(t)\right)$.

An inverse limit space $\lim _{\leftarrow} X_{i}$ is not affected if one disregards a finite number of indices. Lemma 11 therefore applies more generally for any finite choice of coordinates of $y \in M_{\infty}$. It follows that path components are dense.

Lemma 12. Suppose that $M_{\infty}$ is a weak solenoid and that $x_{0}, x_{1} \in M_{\infty}$ are in the same path-component. Then there exists a homeomorphism $f: M_{\infty} \rightarrow M_{\infty}$ such that $f\left(x_{0}\right)=x_{1}$.

Proof. Suppose that $\gamma=\left(\gamma_{i}\right):[0,1] \rightarrow M_{\infty}$ connects $x$ and $y$. Define a isotopy $F_{0}: M_{0} \times[0,1] \rightarrow M_{0}$ such that $F_{0}(x, 0)=x$ and such that $F_{0}\left(x_{0}, t\right)=\gamma_{0}(t)$. Define homotopies $F_{i}: M_{i} \times[0,1] \rightarrow M_{0}$ by $F_{i}=F_{0} \circ\left(p_{0}^{i} \times i d_{[0,1]}\right)$. By the homotopy 
lifting property there exist lifts $\tilde{F}_{i}: M_{i} \times[0,1] \rightarrow M_{i}$ such that $\tilde{F}_{i}\left(x_{i}, t\right)=\gamma_{i}(t)$. By Theorem 8 these lifts commute with the bonding maps of the inverse limit. Hence, the transformation $F: M_{\infty} \rightarrow M_{\infty}$ defined by $F\left(\left(z_{i}\right)\right)=\left(\tilde{F}_{i}\left(z_{i}, 1\right)\right)$ is well defined, it is invertible, and it maps $x$ to $y$.

It follows that a weak solenoid is homogeneous if and only if it is possible to permute path components transitively.

Theorem 13 (McCord, 15]). A strong solenoid is homogeneous.

Proof. Suppose that $\lim _{\leftarrow}\left(M_{i}, p^{i}\right)$ is a solenoid and that $x, y \in M_{\infty}$. By the previous lemmas we may assume that $x_{0}=y_{0}$. By Theorem 9, the regularity of the covering map $p_{0}^{i}:\left(M_{i}, x_{i}\right) \rightarrow\left(M_{0}, x_{0}\right)$ implies that there exists a lift $f_{i}:\left(M_{i}, x_{i}\right) \rightarrow\left(M_{i}, y_{i}\right)$. By Theorem 8 the lifts commute with the bonding maps: $f_{j} \circ p_{j}^{i}=p_{j}^{i} \circ f_{i}$. Hence the map $f: M_{\infty} \rightarrow M_{\infty}$ defined by the $f_{i}$ is invertible and $f(x)=y$.

Consider a solenoid as a bundle over a manifold $p: M_{\infty} \rightarrow M_{0}$. The proof of Theorem 13 implies that for any two points $x, y$ in the same fiber, there exists a transformation $h: M_{\infty} \rightarrow M_{\infty}$ that leaves all the fibers invariant and maps $x$ onto $y$. In this sense, strong solenoids are generalizations of regular coverings.

\section{Group Chains And the Base-Point Fiber}

A covering space of a manifold $M$ corresponds to a subgroup of the fundamental group $\pi_{1}(M)$. Conversely, every subgroup of $\pi_{1}(M)$ corresponds to a covering space. We shall call a descending chain of groups $G_{0} \supset G_{1} \supset G_{2} \supset \ldots$ a group chain. A weak solenoid $\lim _{\leftarrow}\left(M_{i}, m_{i}\right)$ corresponds to a group chain $\pi_{1}\left(M_{i}, m_{i}\right)$. In this terminology, McCord's criterion for homogeneity in [15] is that the group chain consists of normal subgroups from some index onwards. This is not a necessary condition for homogeneity. One can construct group chains $N_{i}$ and $G_{i}$, such that the $N_{i}$ are normal subgroups and the $G_{i}$ are not, while $N_{i+1} \subset G_{i} \subset N_{i}$ [20]. A weak solenoid that corresponds to the group chain $G_{i}$ then is homeomorphic to a strong solenoid that corresponds to the group chain $N_{i}$.

There is an ambiguity in the correspondence between weak solenoids and group chains, since the groups $\pi_{1}\left(M_{i}, m_{i}\right)$ depend on the choice of $m_{i}$. For a proper analogy one needs to consider all possible choices of $m_{i}$. For any weak solenoid $M_{\infty}$ we shall fix a base-point $m_{\infty} \in M_{\infty}$ with coordinates $m_{i} \in M_{i}$. We define the basepoint fiber $B_{\infty} \subset M_{\infty}$ as the subset of all elements $x_{i} \in M_{\infty}$ with $x_{0}=m_{0}$. The base-point fiber is a Cantor set. It is the inverse limit over the fibers $p_{i}^{-1}\left(m_{0}\right) \subset M_{i}$. The fiber $p_{i}^{-1}\left(m_{0}\right)$ is represented by the (right) residue classes of $\pi_{1}\left(M_{i}, m_{i}\right)$ in $\pi_{1}\left(M_{0}, m_{0}\right)$. The base-point fiber is therefore an inverse limit over (right) residue classes. For a strong solenoid, the residue classes are finite and the limit is called a profinite group.

The base-point fiber represents all possible choices of base-points in $M_{i}$ once we have fixed a base point in $M_{0}$. In the same way, we may consider all possible choices of group chains. For elements in the fiber $x, y \in p_{i}^{-1}\left(m_{0}\right)$, the fundamental groups $\pi_{1}\left(M_{i}, x\right)$ and $\pi_{1}\left(M_{i}, y\right)$ are conjugate (under identification in $\pi_{1}\left(M_{0}, m_{0}\right)$ ).

Definition 14. Suppose that $G$ is a group and that $G_{i}$ is a group chain with $G_{0}=G$. Let $\mathcal{K}$ be the collection of all group chains $H_{i}$ such that $H_{i}=g_{i}^{-1} G_{i} g_{i}$ for some sequence $g_{i} \in G$ with the property that $G_{i} g_{j}=G_{i} g_{i}$ for each $j \geq i$. We 
say that $\mathcal{K}$ is a class of conjugate group chains, or, for short, we say that $\mathcal{K}$ is the conjugacy class of $G_{i}$.

We silently assume that group chains have a common universe, i.e., there exists a group $G$ such that each chain consists of subgroups $G_{i} \subset G$ of finite index. The base-point fiber $B_{\infty}$ and the conjugacy class $\mathcal{K}$ both are representations of $\lim _{\leftarrow} G_{0} / G_{i}$. For a conjugacy class $\mathcal{K}$ of a group chain $G_{i}$, denote by $\mathcal{K}_{k}$ the subclass of all chains $H_{i}$ that are conjugate to $G_{i}$ and such that $G_{j}=H_{j}$ for $j \leq k$. Subclasses correspond to a basis of neighborhoods for $m_{\infty} \in B_{\infty}$.

Proposition 15. Suppose that $M_{\infty}$ is a weak solenoid with base-point fiber $B_{\infty}$. The subsets $B_{i_{0}}=\left\{x_{i} \in B_{\infty} \mid x_{j}=m_{j}\right.$ if $\left.j \leq i_{0}\right\}$ form a neighborhood basis of $m_{\infty}$ that corresponds to the subclasses $\mathcal{K}_{i_{0}} \subset \mathcal{K}$.

The fundamental group $\pi_{1}\left(M_{0}, m_{0}\right)$ acts on the fiber of $p_{i}: M_{i} \rightarrow M_{0}$ for every index $i$, and, by taking the inverse limit, it acts continuously on the base-point fiber.

Two group chains $G_{i}$ and $H_{i}$ are called equivalent if for every $i$ there exists a $j$ such that $G_{j} \subset H_{i}$ and $H_{j} \subset G_{i}$.

Definition 16. We shall say that a conjugacy class of group chains $\mathcal{K}$ is weakly normal if for some index $i$ all group chains in the subclass $\mathcal{K}_{i}$ are equivalent.

We shall show that a weak solenoid is homogeneous if and only if its associated class of group chains is weakly normal.

Lemma 17. Suppose that $\left(M_{\infty}, m_{\infty}\right)$ and $\left(N_{\infty}, n_{\infty}\right)$ are weak solenoids that agree on the first coordinate, i.e., $M_{0}=N_{0}$ and $m_{0}=n_{0}$. Suppose that the group chains $G_{i}$ and $H_{i}$, associated to $\left(M_{\infty}, m_{\infty}\right)$ and $\left(N_{\infty}, n_{\infty}\right)$, respectively, are equivalent. Then there exists a base-point-preserving homeomorphism $h:\left(M_{\infty}, m_{\infty}\right) \rightarrow$ $\left(N_{\infty}, n_{\infty}\right)$.

Proof. This is a repeated application of Theorem 9

Recall that the core of a subgroup $H \subset G$ is defined as the intersection of all conjugacy classes of $H$. It is the maximal normal subgroup in $H$.

Theorem 18. Suppose that $M_{\infty}$ is a weak solenoid and that its associated group class is weakly normal. Then $M_{\infty}$ is homeomorphic to a strong solenoid.

Proof. Let $G_{i}$ be the group chain associated to $\left(M_{\infty}, m_{\infty}\right)$. By disregarding first indices we may assume that the conjugacy class of $G_{i}$ is weakly normal. It suffices to show that the chain core $G_{i}$ is equivalent to $G_{i}$, i.e., for every $i_{0}$ there is a $j>i_{0}$ such that $G_{j} \subset$ core $G_{i_{0}}$. Since the index is finite, there are finitely many conjugacy classes of $G_{i_{0}}$ in $G_{0}$, say $G_{i_{0}}^{g_{1}}, \ldots, G_{i_{0}}^{g_{k}}$. By the normality of the class, the chains $G_{i}^{g_{1}}, \ldots, G_{i}^{g_{k}}$ are equivalent to the chain $G_{i}$. Hence there exists a $j \geq i_{0}$ such that $G_{j} \subset G_{i_{0}}^{g_{1}} \cap \ldots \cap G_{i_{0}}^{g_{k}}$, i.e., $G_{j}$ is contained in the core of $G_{i_{0}}$.

If the conjugacy class of a group chain $G_{i}$ is normal, then the group chain is equivalent to core $G_{i}$. All group chains then share the same kernel. For a weakly normal class we have the following result.

Theorem 19. Suppose that a conjugacy class of group chains $\mathcal{K}$ is weakly normal. Then the kernels of the group chains in $\mathcal{K}$ form a finite collection of conjugate groups. 


\section{Proof OF THE MAIN THEOREM}

Proposition 20. Suppose that $M$ is a manifold and that $X$ is an arbitrary topological space. There exists an $\epsilon>0$ such that any two maps $f, g: X \rightarrow M$ that are $\epsilon$-close are homotopic.

Proof. Recall that we only consider compact closed manifolds. A compact closed manifold is an ANR; hence nearby maps are homotopic.

Our proof of Theorem 3 involves a property of inverse limit spaces that is sometimes referred to as Mioduszewski's theorem [17. It applies to homeomorphisms between $\lim _{\leftarrow}\left(P_{i}, f_{i}\right)$ and $\lim _{\leftarrow}\left(Q_{i}, g_{i}\right)$, where the $P_{i}$ and $Q_{i}$ are polyhedra. The property says that any homeomorphism between such spaces can be approximated by a zigzag sequence of maps that almost commute with the bonding maps, as sketched in the diagram below:

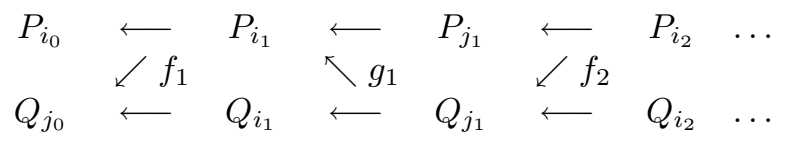

We include a precise statement of this property, stated for our case of pointed spaces. For completeness we also include a proof.

Lemma 21. Suppose that $h: \lim _{\leftarrow}\left(M_{i}, p^{i}\right) \rightarrow \lim _{\leftarrow}\left(N_{i}, q^{i}\right)$ is a homeomorphism between weak solenoids such that $h(m)=n$. For every sequence $\epsilon_{n}$ converging to 0 , there exist an infinite sequence of maps $f_{n}:\left(M_{i_{n}}, m_{i_{n}}\right) \rightarrow\left(N_{j_{n-1}}, n_{j_{n-1}}\right)$ and an infinite sequence of maps $g_{n}:\left(N_{j_{n}}, n_{j_{n}}\right) \rightarrow\left(M_{i_{n}}, m_{i_{n}}\right)$ such that $f_{n} \circ g_{n}$ is $\epsilon_{j_{n-1}}$ close to $q_{j_{n-1}}^{j_{n}}: N_{j_{n}} \rightarrow N_{j_{n-1}}$ and $g_{n} \circ f_{n+1}$ is $\epsilon_{i_{n}}$-close to $p_{i_{n}}^{i_{n+1}}: M_{i_{n+1}} \rightarrow M_{i_{n}}$.

Proof. We choose $j_{0}=0$. Consider $M_{\infty}$ as a subspace of the product $\prod_{i} M_{i}$. Identify $M_{i}$ with the subset of $\prod_{i} M_{i}$ given by

$$
\left\{\left(x_{n}\right) \mid x_{i} \in M_{i}, x_{n}=p_{n}^{i}\left(x_{i}\right) \text { if } n \leq i, x_{n}=m_{n} \text { if } n>i\right\} .
$$

Let $\pi_{i}$ denote the projection on the $i$-th coordinate of a weak solenoid. Observe that $M_{i_{1}}$ is the projection of $M_{\infty}$ under $\pi_{i_{1}}$. Furthermore, for $i_{1}$ sufficiently large, $M_{i_{1}}$ is contained in any given neighborhood of $M_{\infty}$ in $\prod_{i} M_{i}$. Consider the composition $\pi_{0} \circ h:\left(M_{\infty}, m\right) \rightarrow\left(N_{0}, n_{0}\right)$. Since $N_{0}$ is an ANR, there exist a neighborhood $U$ of $M_{\infty}$ in $\prod_{i} M_{i}$ and an extension $\tilde{f}_{1}: U \rightarrow N_{0}$ such that $\tilde{f}_{1}\left(m_{i}\right)=n_{i}$. Define $f_{1}:\left(M_{i_{1}}, m_{i_{1}}\right) \rightarrow\left(N_{0}, n_{0}\right)$ as the restriction of $\tilde{f}_{1}$. We may choose $i_{1}$ sufficiently large so that $\pi_{0} \circ h$ and $f_{1} \circ \pi_{i_{1}}$ are $\epsilon_{0} / 2$ close. This is our first map in the zigzag sequence.

We repeat the construction for the inverse of the homeomorphism $h$. Choose $\delta_{0}$ such that for any two points in $M_{i_{1}}$ which are $\delta_{0}$ close the images under $f_{1}$ are $\epsilon_{0} / 2$ close. Extend the composition $\pi_{i_{1}} \circ h^{-1}:\left(N_{\infty}, n\right) \rightarrow\left(M_{i_{1}}, m_{i_{1}}\right)$ to $\tilde{g}_{1}: V \rightarrow M_{i_{1}}$, where $V$ is a neighborhood of $N_{\infty}$ in the product space $\prod_{j} N_{j}$ and $\tilde{g}_{1}\left(n_{i}\right)=m_{i_{1}}$. For $j_{1}$ sufficiently large, $N_{j_{1}} \subset V$. Define $g_{1}:\left(N_{j_{1}}, n_{j_{1}}\right) \rightarrow\left(M_{i_{1}}, m_{i_{1}}\right)$ as the restriction of $\tilde{g}_{1}$. This is the second map of our zigzag sequence. Let $N_{j_{1}}$ be the projection of $N_{\infty}$ on the $j_{1}$-th coordinate. By choosing $j_{1}$ sufficiently large, we may suppose that $\pi_{i_{1}} \circ h^{-1}$ and $g_{1} \circ \pi_{j_{1}}$ are $\delta_{0}$-close. Now for any $x \in N_{j_{1}}$ suppose that $x_{\infty} \in N_{\infty}$ is an element such that its $j_{1}$-th coordinate is $x$. By our conditions $g_{1}(x)$ and $\pi_{1} \circ h^{-1}\left(x_{\infty}\right)$ are $\delta_{0}$-close. Hence $f_{1} \circ g_{1}(x)$ and $f_{1} \circ \pi_{i_{1}} \circ h^{-1}\left(x_{\infty}\right)$ are $\epsilon_{0} / 2$-close. By our conditions 
on $i_{1}$ we have that $f_{1} \circ \pi_{i_{1}} \circ h^{-1}\left(x_{\infty}\right)$ and $\pi_{0} \circ h \circ h^{-1}\left(x_{\infty}\right)=q_{0}^{j_{1}}(x)$ are $\epsilon_{0} / 2$ close. Hence $f_{1} \circ g_{1}$ is $\epsilon_{0}$-close to $q_{0}^{j_{1}}$. Continue the construction inductively.

Another element of the proof is Effros' Theorem [9]. Recall that for a compact space $X$, the space of homeomorphisms $h: X \rightarrow X$ is metrizable.

Definition 22. A metric space $(X, d)$ is micro-homogeneous if for every $\epsilon>0$ there exists a $\delta>0$ such that if $d(x, y)<\delta$ then there is a homeomorphism $h:(X, x) \rightarrow(X, y)$ such that $d\left(h, i d_{X}\right)<\epsilon$.

Theorem 23 (Effros). If a compact metric space is homogeneous, then it is microhomogeneous.

In the proof of the next lemma we shall use the fact that projection onto a coordinate is non-expansive under the natural product metric on a weak solenoid.

Lemma 24. Suppose that $M_{\infty}$ is a homogeneous weak solenoid. There exists a $\delta>0$ such that for any pair $p, q \in M_{\infty}$ with equal first coordinates and with $d(p, q)<\epsilon$, there exists a homeomorphism $h: M_{\infty} \rightarrow M_{\infty}$ which maps $p$ onto $q$ and which leaves the first coordinate of $M_{\infty}$ invariant.

Proof. Fix $\epsilon>0$ such that self-maps of $M_{\infty}$ that are $\epsilon$-close are homotopic. By Effros' Theorem, if $d(p, q)<\delta$ then there exists a homeomorphism such that $h(p)=$ $q$ and $d(h, i d)<\epsilon$. Let $h_{0}$ be the projection of $h$ onto the first coordinate. It is $\epsilon$-close to the projection $p_{0}: M_{\infty} \rightarrow M_{0}$. By our choice of $\epsilon$ there exists a homotopy $H: M_{\infty} \times[0,1] \rightarrow M_{0}$ such that $H(x, 0)=h_{0}(x)$ and $H(x, 1)=x_{0}$. The map $h_{0}$ can be lifted to $M_{1}$, since it is a projection from $M_{\infty}$. By the homotopy lifting property, $H$ can be lifted to $\tilde{H}: M_{1} \times I \rightarrow M_{1}$. Inductively we get a homotopy $\tilde{H}: M_{\infty} \times[0,1] \rightarrow M_{\infty}$, and the map $\tilde{H}(x, 1)$ has the desired property.

There is a technicality that we have ignored so far. We have associated a weak solenoid $\left(M_{\infty}, m\right)$ to a chain of groups $G_{i}=\pi_{1}\left(M_{i}, m_{i}\right)$, but the $G_{i}$ are not subgroups of $G_{0}$. They are embedded in $G_{0}$ by the monomorphisms $p_{*}^{i}: G_{i} \rightarrow G_{0}$. We shall now be a little more precise.

Theorem 25. If a generalized weak solenoid is homogeneous, then its associated group chain is weakly normal.

Proof. Suppose that $\left(M_{\infty}, m_{\infty}\right)$ is a homogeneous weak solenoid, and that $\epsilon_{i}$ is a sequence of positive numbers associated to $M_{i}$ as in Proposition [20] For every $x \in B_{\infty}$ there exists a homeomorphism $h_{x}: M_{\infty} \rightarrow M_{\infty}$ such that $h_{x}\left(m_{\infty}\right)=x$. By the previous lemma there exists a neighborhood $V \subset B_{\infty}$ such that for each $x \in V$ we may assume that $h_{x}$ leaves the first coordinate invariant. We shall show that all group chains associated to elements of $V$ are equivalent. By Proposition 15 this implies that the group chain is weakly normal.

By Lemma 21 there exist sequences of maps $f_{n}:\left(M_{i_{n}}, m_{i_{n}}\right) \rightarrow\left(M_{j_{n-1}}, x_{j_{n-1}}\right)$ and $g_{n}:\left(M_{j_{n}}, x_{i_{n}}\right) \rightarrow\left(M_{i_{n}}, m_{i_{n}}\right)$, which give a zigzag sequence

$$
\left(M_{0}, x_{0}\right) \stackrel{f_{1}}{\longleftarrow}\left(M_{i_{1}}, m_{i_{1}}\right) \stackrel{g_{1}}{\longleftarrow}\left(M_{j_{1}}, x_{j_{1}}\right) \stackrel{f_{2}}{\longleftarrow} \ldots
$$

Since the zigzag is induced by a map that leaves the first coordinate invariant, we may extend it by $g_{0}:\left(M_{0}, x_{0}\right) \rightarrow\left(M_{0}, m_{0}\right)$ equal to the identity. The resulting zigzag is $\epsilon_{i}$-almost commutative. 
Let $G_{i}$ be the group chain associated to $m$ and let $H_{i}$ be the group chain associated to $x$. We claim that the zigzag implies that $H_{j_{n}} \subset G_{i_{n}} \subset H_{j_{n-1}}$, which implies that the conjugacy class is normal. Observe that by the choice of the $\epsilon_{i}$ the induced zigzag $f_{n_{*}}: G_{i_{n}} \rightarrow H_{j_{n-1}}$ and $g_{n_{*}}: H_{j_{n}} \rightarrow G_{i_{n}}$ commutes with $p_{i_{n-1 *}}^{i_{n}}$ and $p_{j_{n-1 *}}^{j_{n}}$. Hence $p_{*}^{j_{n-1}} \circ f_{n_{*}}\left(G_{i_{n}}\right)=g_{0_{*}} \circ p_{*}^{i_{n}}\left(G_{i_{n}}\right)=p_{*}^{i_{n}}\left(G_{i_{n}}\right)$. Now it is time to recall that the $G_{i_{n}}$ are actually defined by the monomorphism $p_{*}^{i_{n}}$ that maps $\pi_{1}\left(M_{i_{n}}, m_{n}\right)$ into $\pi_{1}\left(M_{0}, m_{0}\right)$. So we should read $p^{j_{n-1}} \circ f_{n_{*}}\left(\pi_{1}\left(M_{i_{n}}, m_{i_{n}}\right)\right)=G_{i_{n}}$. We also have that $p^{j_{n-1}}{ }_{*}^{\circ} \circ f_{n_{*}}\left(\pi_{1}\left(M_{i_{n}}, m_{i_{n}}\right)\right) \subset p^{j_{n-1}}{ }_{*}\left(\pi_{1}\left(M_{j_{n-1}}, x_{j_{n-1}}\right)\right)=H_{j_{n-1}}$. The inclusion $G_{i_{n}} \subset H_{j_{n-1}}$ now follows. The other inclusion follows by symmetry.

Theorem 3 now follows from Theorem 18 and Theorem 25

\section{The Schori and Rogers-Tollefson examples Revisited}

We shall now study the examples of non-homogeneous weak solenoids by Schori 22 and by Rogers and Tollefson [19], to illustrate our results. We know that the group chain of a homogeneous weak solenoid is interlaced with a normal chain. It follows that for a homogeneous solenoid, all group chains in the conjugacy class have the same core. Schori essentially shows that if a weak solenoid $M_{\infty}$ has a group chain $G_{i}$ with a kernel that has infinitely many conjugacy classes, then $M_{\infty}$ is not homogeneous. He then explicitly constructs $M_{\infty}$. One can also obtain this result by using machinery from geometric group theory.

Definition 26. A group $G$ is geometrically residually finite if every subgroup $H \subset$ $G$ is equal to the kernel of a group chain $G_{i}$ of finite index.

Theorem 27 (Scott, 23]). The fundamental group of a closed surface is geometrically residually finite.

If a closed surface has negative Euler characteristic, then the fundamental group has at least three generators and only one relation, containing all generators. Schori's example now follows from the following observation.

Proposition 28. The fundamental group of a hyperbolic surface contains an infnite cyclic subgroup with infinitely many conjugate subgroups.

Proof. Suppose that $x_{1}$ is one of the generators of the fundamental group. If the infinite cyclic group $\left\langle x_{1}\right\rangle$ has only finitely many conjugacy classes, then $x_{1}$ commutes with some iterate $x_{2}^{k}$. However, there is no relation between $x_{1}$ and $x_{2}$ only.

Rogers and Tollefson construct a non-homogeneous weak solenoid as an inverse limit space over Klein bottles. Their original argument for non-homogeneity is that one of the path components is non-orientable while all other components are orientable. We shall study their example in terms of group chains.

The fundamental group of the Klein bottle $\mathcal{B}$ has two generators and one relation, $\pi_{1}(\mathcal{B})=\left\langle a, b: b a b^{-1}=a^{-1}\right\rangle$. The torus $\mathcal{T}$ is a double cover of the Klein bottle, that corresponds to the abelian subgroup generated by $a$ and $b^{2}$. Represent the torus as $\mathbb{R} / \mathbb{Z} \times \mathbb{R} / \mathbb{Z}$ and the Klein bottle as its quotient under $i:(x, y) \rightarrow\left(x+\frac{1}{2},-y\right)$. The linear map $L: \mathcal{T} \rightarrow \mathcal{T}$ given by $L(x, y)=(x, 2 y)$ is a double cover of the torus by itself. Since $L$ and $i$ commute, $L$ induces a double cover of the Klein bottle by itself $p: \mathcal{B} \rightarrow \mathcal{B}$. The non-homogeneous solenoid $\mathcal{B}_{\infty}$ of Rogers and Tollefson is the inverse limit over $p$. It is a quotient space of the cartesian product of a circle and the standard 2-solenoid, under a fixed-point free involution. 
Since $p: \mathcal{B} \rightarrow \mathcal{B}$ is a 2 -to- 1 map, it is a regular covering. The induced homomorphism $p_{*}: \pi_{1}(\mathcal{B}) \rightarrow \pi_{1}(\mathcal{B})$ is given by $p_{*}(a)=a^{2}$ and $p_{*}(b)=b$. The composition $p^{2}$ is not a regular covering map since the subgroup $\left\langle a^{4}, b\right\rangle$ is not normal. Indeed, it is conjugated to $\left\langle a^{4}, a^{2} b\right\rangle$ under conjugation by $a$. A group chain $G_{i}$ associated to $\mathcal{B}_{\infty}$ is of the form $G_{i}=\left\langle a^{2^{i}}, a^{k_{i}} b\right\rangle$ for a sequence $k_{i+1}=k_{i} \bmod 2^{i}$. Since $G_{i}$ contains $b^{2}$, the kernel of each such chain contains the normal subgroup $\left\langle b^{2}\right\rangle$. We shall show that the kernel of a chain is either equal to $\left\langle b^{2}\right\rangle$, or conjugate to $\langle b\rangle$, which is not a normal subgroup. This implies that the solenoid is not homogeneous.

Denote the normal subgroup $\pi_{1}(\mathcal{T})$ by $N=\left\langle a, b^{2}\right\rangle$. For a group chain $G_{i}$ let $N_{i}$ be the chain given by $N_{i}=G_{i} \cap N$, Observe that $N_{i}=\left\langle a^{2^{i}}, b^{2}\right\rangle$ is normal and independent of the choice of the group chain $G_{i}$. Let $N_{\infty}$ be the kernel of $N_{i}$, and $G_{\infty}$ the kernel of $G_{i}$. As the index $\left[G_{i}: N_{i}\right] \leq 2$ and as $N_{\infty}$ is a normal subgroup, one verifies that $G_{\infty} / N_{\infty}$ is a group of at most order two.

Let $H$ be the subgroup of $\pi_{1}(\mathcal{B})$ generated by $b$ and let $N$ be the normal subgroup generated by $a$. Then $\pi_{1}(\mathcal{B})$ is the semi-direct product of $H$ and $N$. The action of $H$ on $N$ is prescribed by the one relation, $b a b^{-1}=a^{-1}$. Each element can be represented uniquely as $a^{n} b^{m}$ for $n, m \in \mathbb{Z}$. One verifies that the following is true.

Proposition 29. Suppose that $H=\left\langle a^{2^{i}}, a^{k_{i}} b\right\rangle$ is a subgroup of $\pi_{1}(\mathcal{B})$. Then each element of $H$ can be represented uniquely as $a^{n} b^{m}$ with $n=0 \bmod 2^{i}$ if $m$ is even and $n=k \bmod 2^{i}$ if $m$ is odd.

This proposition implies that the kernel of the group chain is equal to $\left\langle b^{2}\right\rangle$ unless the sequence $k_{i}$ stabilizes. In that case the group chain is conjugate to the chain $\left\langle a^{2^{i}}, b\right\rangle$, which has kernel $\langle b\rangle$. This implies that the weak solenoid $\mathcal{K}_{\infty}$ is not homogeneous.

\section{WeAK SOlENOIDS ARE $G$-BUNDLES}

One-dimensional solenoids fiber over the circle. The standard dyadic solenoid, for instance, is a principal $\mathbb{Z}_{2}$-bundle, where $\mathbb{Z}_{2}$ denotes the group of 2-adic integers. This characterization extends to generalized solenoids, the weak solenoids being $\Gamma$-bundles and the strong solenoids being principal $\Gamma$-bundles, for a profinite group $\Gamma$.

Definition 30. Suppose that $G$ is a group and that $N_{i}$ is a descending chain of normal subgroups of finite index. The inverse limit $\Gamma=\lim _{\leftarrow} G / N_{i}$, endowed with the natural topology, is called a profinite group.

A general reference on the subject is [28]. The following characterization is convenient.

Theorem 31. A topological group $\Gamma$ is profinite if and only if it is compact, zerodimensional and there exists a descending chain $\Gamma \supset H_{1} \supset H_{2} \supset \ldots$ of open subgroups with kernel $\{e\}$.

Recall that a fiber bundle $p: Y \rightarrow B$ is a principal $G$-bundle if the fibers are homeomorphic to $G$ and the transition maps are induced by $G$ translations, see 25]. The following observation is due to McCord [15].

Theorem 32. A strong solenoid is a principal $\Gamma$-bundle for a profinite group $\Gamma$. 
Proof. The base-point fiber of a strong solenoid $\left(M_{\infty}, m_{\infty}\right)$ has the natural structure of a profinite group. For a simply connected neighborhood $m_{0} \in U \subset M_{0}$, consider the open subset $U_{\infty}=\left\{x \in M_{\infty} \mid x_{0} \in U\right\}$ and the natural chart $p_{U, m_{\infty}}: U_{\infty} \rightarrow U \times B_{\infty}$. Note that the chart depends on the choice of the basepoint. A different choice of the base-point corresponds to a translation $h \rightarrow g \circ h$ of the base-point fibre. Thus, transition maps between charts are $\Gamma$-translations.

Weak solenoids are bundles, though not necessarily principal.

Theorem 33. A weak solenoid is a $\Gamma$-bundle for a profinite group $\Gamma$.

Proof. As for the choice of the charts, there is no difference with strong solenoids. However, the base-point fiber of a weak solenoid may not admit a group structure. Suppose that $M_{\infty}$ is a weak solenoid with group chain $G_{i}$. The normal chain core $G_{i}$ gives an inverse limit $\Gamma=\lim _{\leftarrow} G_{0} /\left(\operatorname{core} G_{i}\right)$ that is a profinite group, which acts on $\lim _{\leftarrow} G_{0} / G_{i}$ by right multiplication. Thus the weak solenoid is a $\Gamma$-bundle.

\section{WEAK SOlENOIDS WITH SIMPLY CONNECTED PATH COMPONENTS}

Rogers and Tollefson posed the following question in [20].

Question 34. Is a weak solenoid homogeneous if all its path-components are homeomorphic?

It is not easy to decide whether path components of weak solenoids are homeomorphic. In the one-dimensional case, Ronald de Man has shown that path components of solenoids are homeomorphic, even if the solenoids are not themselves homeomorphic 13. We shall not settle this question, but we shall give the following example that, as indicated by De Man's result, is a good candidate for a negative answer.

Theorem 35. There exists a weak solenoid that is non-homogeneous but all of its path components are simply connected.

The construction of such a weak solenoid is an algebraic problem.

Lemma 36. There exists a non-homogeneous weak solenoid $M_{\infty}$ with simply connected path-components if and only if there exists a group chain $G_{i}$ with conjugacy class $\mathcal{K}$ such that

(a) $G_{0}$ is finitely presented,

(b) the kernel of all group chains in $\mathcal{K}$ is $\{e\}$, and

(c) $\mathcal{K}$ is not weakly normal.

Proof. We know that a weak solenoid $M_{\infty}$ with simply connected path-components has a group chain $G_{i}$ with the given algebraic properties. Conversely, for every finitely presented group $G$ there exists a closed manifold with fundamental group $G$, see e.g. 14. Hence the existence of a group chain $G_{i}$ that satisfies the conditions (a), (b), (c) implies the existence of a weak solenoid that is non-homogeneous and has simply connected path components.

Hendrik Lenstra constructed a group chain that satisfies the three algebraic conditions (a), (b), (c), during one of his swims. The key idea in his construction is to translate the rather awkward conditions on group chains of Lemma 36 into elegant conditions on subgroups of profinite groups. 
Lemma 37 (Lenstra). Suppose that $\Gamma$ is a profinite group with a closed subgroup $H \subset \Gamma$ that has infinitely many conjugacy classes. Suppose that $G \subset \Gamma$ is a finitely presented, dense subgroup that intersects each conjugation class of $H$ in $\{e\}$. Then there exists a group chain that satisfies (a), (b), (c) of Lemma[36.

Proof. Since $H$ is a closed subgroup, there exists a descending chain of subgroups $H_{i}$ of finite index in $\Gamma$ and kernel $H=\bigcap H_{i}$. Then $G_{i}=G \cap H_{i}$ is a group chain with kernel $G \cap H=\{e\}$. Since $G$ is finitely presented, it satisfies condition (a) of Lemma 36. Suppose that $G_{i}^{g_{i}}$ is a conjugate chain. To satisfy condition (b), its kernel has to intersect $H$ in $\{e\}$. For every $i$ we have $g_{i+1} G_{i}=g_{i} G_{i}$, and by the density of $G$ it follows that $g_{i+1} H_{i}=g_{i} H_{i}$. The chain $g_{i} H_{i}$ is descending, and by compactness its kernel $\bigcap g_{i} H_{i}$ is non-empty. For any element $h$ in the kernel, the chain $G_{i}^{g_{i}}$ is equal to $G_{i}^{h}$. Since $G$ intersects every conjugacy class of $H$ in $\{e\}$, the kernel of $G_{i}^{h}$ is equal to $\{e\}$, and condition (b) is satisfied.

We verify that the chain $G_{i}$ satisfies condition (c) by contradiction. Suppose that $\mathcal{K}$ is weakly normal, i.e., for some index $k$ all conjugate chains $G_{i}^{g_{i}}$ with $g_{i}=e$ for $i \leq k$ are equivalent to $G_{i}$. In the profinite group, the elements with $g_{i}=e$ for $i \leq k$ form an open subgroup $V \subset \Gamma$. For every $\gamma \in V$ the chains $\left(G \cap H_{i}\right)^{\gamma}$ and $G \cap H_{i}$ are equivalent; hence they have the same kernel. By the density of $G$, $H=H^{\gamma}$ for every $\gamma \in V$. Since $V$ has finite index, this contradicts the fact that $H$ has infinitely many conjugacy classes.

With this translation in hand, one may construct various group chains satisfying the conditions of Lemma 36. Recall that an (external) semi-direct product $G=$ $H \ltimes N$ is induced by a group homomorphism $\alpha: H \rightarrow \operatorname{Aut}(N)$, and that every element of $G$ can be represented uniquely as $h n$ for $h \in H$ and $n \in N$. For profinite groups, the automorphism group $\operatorname{Aut}(N)$ is endowed with the topology of uniform convergence. If the group homomorphism $\alpha: H \rightarrow \operatorname{Aut}(N)$ is continuous, then the natural topology turns $H \ltimes N$ into a profinite group.

Proposition 38. Suppose that $H, N$ are (non-discrete) profinite group and that $\alpha: H \rightarrow \operatorname{Aut}(N)$ is a continuous group homomorphism. If for every $n \in N \backslash\{e\}$ there exists an $h \in H$ such that $\alpha(h)(n) \neq n$, then $\Gamma=H \ltimes N$ is a profinite group and $H$ has infinitely many conjugacy classes.

Proof. Let $N_{i} \subset N$ be a chain of open normal subgroups. By the continuity of $\alpha$, there is a corresponding chain $H_{i} \subset H$ of open subsets such that $\alpha\left(h_{i}\right)\left(N_{i}\right)=N_{i}$ for all $h_{i} \in H_{i}$. In $H \ltimes N$, the subgroups $H_{i} N_{i}$ are open and have intersection $\{e\}$. By Theorem 31, $H \ltimes N$ is a profinite group.

Suppose that $n \in N$ and that $H^{n}=H$ in $H \ltimes N$. Since $n h n^{-1}=h(\alpha(h)(n)) n \in$ $H$, and by the uniqueness of the representation, $\alpha(h)(n)=n$ for every $h \in H$. Hence, the normalizer $\left\{n \in N: H^{n}=H\right\}$ coincides with the subgroup $\{n \in$ $N: \forall h \in H, \alpha(h)(n)=n\}$. This subgroup is trivial provided that for every $n \in N \backslash\{e\}$ there exists an $h \in H$ such that $\alpha(h)(n) \neq n$. As $N$ is infinite, $H$ has infinitely many conjugacy classes.

We denote the action of $H$ on $N$ by $n^{h}$.

Lemma 39. There exist a profinite group $\Gamma$ and subgroups $G, H \subset \Gamma$ that satisfy the conditions of Lemma 37 .

Proof. Let $C=\{e, h\}$ be the cyclic group of order two and let $\mathbb{Z}_{p}$ denote the additive group of $p$-adic integers. Then $C$ acts freely on $\mathbb{Z}_{3}$ under $n^{h}=-n$. By the 
previous proposition $C \ltimes \mathbb{Z}_{3}$ is a profinite group, in which $C$ has infinitely many conjugacy classes. Now consider the product $\Gamma=C \ltimes \mathbb{Z}_{3} \times \mathbb{Z}_{5} \times \mathbb{Z}_{7}$. The subgroup $H=C \times \mathbb{Z}_{5} \times\{0\} \subset \Gamma$ is closed and has infinitely many conjugation classes. The subgroup $N=\mathbb{Z}_{3} \times\{0\} \times \mathbb{Z}_{7}$ is normal. Consider the subgroup $G \subset \Gamma$ generated by $s=(h, 1,1)$ and $t=(1,0,0)$. It is finitely presented: $G \cong\left\langle s, t: s t s^{-1}=t^{-1}\right\rangle$; indeed, it is isomorphic to the fundamental group of the Klein bottle. By the Chinese remainder theorem, $G$ is dense in $\Gamma$.

We need to show that $G$ intersects each conjugation class of $H$ in the identity. Let $s^{n_{1}} t^{m_{1}} s^{n_{2}} t^{m_{2}} \ldots s^{n_{i}} t^{m_{i}}$ an arbitrary element of $G \cap H$. Since the third coordinate of elements of $H$ is zero, the sum $n_{1}+n_{2}+\ldots+n_{i}$ is zero. Since $\Gamma$ is a semi-direct product, we can move all the powers of $s$ to the left, to get an element of the form $s^{n_{1}+n_{2}+\ldots+n_{i}} t^{k}$ for some integer $k$. The exponent of $s$ is zero and $t^{k}$ is contained in $N$. It follows that $G$ intersects each conjugation class of $H$ in the identity.

Proof of Theorem 35. From the previous lemma we obtain a weak solenoid $M_{\infty}$ that is not homogeneous, but has simply connected path components. As we remarked already, the group $G$ in that lemma is isomorphic to the fundamental group of the Klein bottle, $\left\langle s, t: s t s^{-1}=t^{-1}\right\rangle$. A group chain that is not weakly normal, but has kernel $\{e\}$ under every conjugation, is $G_{i}=\left\langle s^{35^{i}}, t^{3^{i}}\right\rangle$. Denote the onedimensional $n$-adic solenoid by $\mathcal{S}_{n}$ and recall that it is a compact abelian group. One may verify that $M_{\infty}$ is a quotient space of the product $\mathcal{S}_{3} \times \mathcal{S}_{35}$ under the involution $(x, y) \rightarrow\left(x+\frac{1}{2},-y\right)$, reminiscent of Rogers and Tollefson's example.

\section{Final Remarks}

A logical next step in the study of weak solenoids would be a study of their selfhomeomorphisms. Rogers and Tollefson showed that the group of auto-homeomorphisms $\mathcal{H}(S)$ determines a solenoid up to homeomorphism. Now $\mathcal{H}(S)$ is a very large group, and it would be interesting to obtain information on the much smaller group of homeomorphisms up to isotopy. A forthcoming paper [7] studies the action of the homeomorphism group on path components of solenoids. The main result of that paper is a criterion for solenoids to be bihomogeneous. Recall that a continuum $K$ is bihomogeneous if for every $x, y \in K$ there is a self-homeomorphism $h$ such that $h(x)=y$ and $h(y)=x$. An example of a homogeneous continuum that is non-bihomogeneous was first constructed by Krystyna Kuperberg [12], satisfying the strong additional condition of local connectivity. Kuperberg has noted that a

subsequent example of Minc [16] can be adapted to construct strong solenoids that are not bihomogeneous.

\section{ACKNOWLEDGEMENT}

We would like to thank Alex Clark for helpful remarks, and Hendrik Lenstra for patiently explaining his algebraic construction and for clarifying our results.

\section{REFERENCES}

[1] Aarts, J.M., Fokkink, R.J., The classification of solenoids, Proc. Amer. Math. Soc. 111, (1991), no. 4, 1161-1163. MR 91g:54046

[2] Aarts, J.M., Hagopian, C., Oversteegen, L.G., The orientability of matchbox manifolds, Pacific J. Math., 150 (1991), no. 1, 1-12. MR 92h:54044

[3] Aarts, J.M., Martens, M., Flows on one-dimensional spaces, Fund. Math. 131 (1988), no. 1, 53-67. MR 89k:54093 
[4] Ancel, F. D., An alternative proof and applications of a theorem of E. G. Effros, Michigan Math. J. 34 (1987), no. 1, 39-55. MR 88h:54058

[5] Bing, R. H., A simple closed curve is the only homogeneous bounded plane continuum that contains an arc, Canad. J. Math. 12 (1960), 209-230. MR 22:1869

[6] Clark, A., A generalization of Hagopian's theorem and exponents, Topology Appl. 117 (2002), no. $3,273-283$.

[7] Clark, A., Fokkink, R.J., Bihomogeneity of solenoids, Algebraic Geom. Topol., 2 (2002), 1-8.

[8] Dantzig, D. van, Waerden, B.L. van der, Über metrisch homogene Räume, Abh. Math. Seminar Hamburg 6, (1928), 367-376.

[9] Effros, E.G., Transformation groups and $C^{*}$-algebras, Ann. of Math (2) 81, (1965), 38-55. MR 30:5175

[10] Hagopian, C. L., A characterization of solenoids, Pacific J. Math. 68 (1977), no. 2, 425-435. MR 56:16584

[11] Keesling, J., The group of homeomorphisms of a solenoid, Trans. Amer. Math. Soc. 172 (1972), 119-131. MR 47:4284

[12] Kuperberg, K., On the bihomogeneity problem of Knaster, Trans. Amer. Math. Soc. 321 (1990), no. 1, 129-143. MR 90m:54043

[13] Man, R. de, On path components of solenoids, Electronic Research Announcement, Amer. Math. Soc. 1 (1995), no. 2, 87-90. MR 96g:54041

[14] Massey, W.S., A basic course in algebraic topology, Springer, New York, 1991. MR 92c:55001

[15] McCord, M.C., Inverse limit sequences with covering maps, Trans. Amer. Math. Soc. 114 (1965), 197-209. MR 30:3450]

[16] Minc, P., Solenoids and bihomogeneity, Continua, Lecture Notes in Pure and Appl. Math., 170, Dekker, New York, 1995, 297-304. MR 96b:54051

[17] Mioduszewski, J. Mappings of inverse limits, Colloq. Math. 10 (1963), 39-44. MR 29:4035

[18] Mislove, M. W., Rogers, J. T., Jr. Local product structures on homogeneous continua, Topology Appl. 31 (1989), no. 3, 259-267. MR 90m:54044

[19] Rogers, Jr., J.T., Tollefson, J.L., Involutions on solenoidal spaces, Fundamenta Math. 73 (1971), 11-19. MR 45:5982

[20] Rogers, Jr., J.T., Tollefson, J.L., Homogeneous inverse limit spaces with nonregular covering maps as bonding maps, Proc. Amer. Math. Soc. 29 (1971), 417-420. MR 42:8439

[21] Rogers, Jr., J.T., Tollefson, J.L., Homeomorphism groups of weak solenoidal spaces, Proc. Amer. Math. Soc. 28 (1971), 242-246. MR 42:6805

[22] Schori, R., Inverse limits and homogeneity, Trans. Amer. Math. Soc. 124 (1966), 533-539. MR 33:6574

[23] Scott, G.P., Subgroups of surface groups are almost geometric, J. London Math. Soc. (2) 17, (1978), no. 3, 555-565. MR 58:12996

[24] Segal, J., Homogeneity of inverse limit spaces, Notices Amer. Math. Soc. 5 (1958), 687.

[25] Steenrod, N., The topology of fibre bundles, Princeton, 1951. MR 12:522b

[26] Ungar, G. S., On all kinds of homogeneous spaces, Trans. Amer. Math. Soc. 212 (1975), 393-400. MR 52:6684

[27] Williams, R. F., Expanding attractors, Inst. Hautes Études Sci. Publ. Math. No. 43, (1974), 169-203. MR 50:1289

[28] Wilson, J.S., Profinite groups, Clarendon Press, Oxford, 1998. MR 2000j:20048

Faculty of Information Technology and Systems, Technische Universiteit Delft, Division Mediamatica, P.O. Box 5031, 2600 GA Delft, Netherlands

E-mail address: r.j.fokkink@its.tudelft.nl

Department of Mathematics, University of Alabama at Birmingham, Birmingham, AlABAMA 35294

E-mail address: overstee@vorteb.math.uab.edu 